\title{
Effects of disorder on two coupled Hubbard chains at half-filling
}

\author{
Satoshi Fujimoto \\ Department of Physics, Kyoto University, Kyoto 606, Japan \\ Norio Kawakami \\ Department of Applied Physics, Osaka University, Suita, Osaka 565, Japan
}

(October 30, 2018)

\begin{abstract}
We investigate the effects of quenched disorder on two chain Hubbard models at half-filling by using bosonization and renormalization group methods. It is found that the sufficiently strong forward scattering due to impurities and the random gauge field, which is generated by impurity backward scattering, destroy the charge gaps as well as the spin gaps. Random backward scattering due to impurities then drives the resulting massless phase to the Anderson localization phase. For intermediate strength of random forward scattering, however, the spin gaps still survive, and only one of the charge gaps is collapsed. In this parameter region, one of the charge degrees of freedom is in the Anderson localized state, while the other one is still in the massive state.
\end{abstract}

\section{INTRODUCTION}

Coupled chain systems with ladder structure have attracted much current interest in the study of strongly correlated electron systems. It has been proposed that these systems could show a superconducting state in the metallic phase.1 1 Such ladder systems have been real ized experimentally in $\mathrm{Sr}_{\mathrm{n}-1} \mathrm{Cu}_{\mathrm{n}+1} \mathrm{O}_{2 \mathrm{n}}$ and $\mathrm{VO}_{2} \mathrm{P}_{2} \mathrm{O}_{7}$. th More recently it has been reported that hole-doped $\mathrm{Sr}_{\mathrm{n}-1} \mathrm{Cu}_{\mathrm{n}+1} \mathrm{O}_{2 \mathrm{n}}$ systems actually undergo the transition to the superconductivity under pressure.6 As microscopic models for such systems, two coupled Hubbard chains and two coupled $t$ - $J$ chains have been extensively studied. 13,15 The ground state of these models at halffilling is the Mott insulator with spin gap. When holes are doped into these systems, a metallic state with spin gap is realized. The resulting massless charge mode belongs to a class of the Tomonaga-Luttinger (TL) liquid (sometimes referred to as the Luther-Emery class), and has the enhanced fluctuation toward superconductivity This superconducting state may be related with that found in $\mathrm{Sr}_{\mathrm{n}-1} \mathrm{Cu}_{\mathrm{n}+1} \mathrm{O}_{2 \mathrm{n}}$.

Such ladder systems are quasi-one-dimensional, so that they may be quite sensitive to random potentials. It is thus important to investigate the effects of quenched disorder on two coupled Hubbard chains. The case away from half-filling was studied by Orignac and Giamarchi before 16 However the case at half-filling has not been considered so far. In this case, we can systematically study how the Mott transition competes with the Anderson localization when the disorder is introduced to the system. This issue was previously addressed by the present authors, but for one-dimensional (1D) interacting electron systems.17(see also refs.18 and 19) In contrast to such single chain models, the Mott insulating state for two coupled Hubbard chains at half-filling has the spin gap as well as the charge gap. Since the randomness affects not only the charge gap but also the spin gap, it is expected that the presence of the spin gap may bring about novel properties, which have not been observed in the single chain model, for the competition between the Mott transition and the Anderson localization

In this paper, we systematically study the effects of disorder on the two coupled Hubbard chains at halffilling. By exploiting bosonization and renormalization group methods, we discuss how the introduction of disorder drives the system to the Anderson localized state. In particular, we focus on the role played by the spin gap for the competition between the Mott insulator and the Anderson localization.

We deal with two types of quenched disorder: random impurity potentials within each band, and random hopping between two bands. We first discuss the effects of the former type of disorder. As was pointed out in ref. 17, the backward scattering due to impurities generates random gauge field coupled with electron currents. We find that the resulting random gauge fields play an essential role for destroying a charge gap in the coupled-chain systems. At half-filling, sufficiently strong random forward scattering due to impurities and random gauge fields destroy charge gaps as well as spin gaps, and the resulting massless charge modes are localized by impurity backward scattering. However, for intermediate strength of random forward scattering, only one of the charge gaps is collapsed, and other modes are still gapful. In this case, we find a parameter region where one charge degrees of freedom is in the Anderson localized state, while the other one is still in the massive state.

In the case of random hopping between two bands, the analysis is more difficult, because two charge modes are mixed by random hopping. However introducing a unitary transformation, we can avoid this difficulty and find the same low-energy fixed point as the case without random interband hopping.

The organization of the paper is as follows: In Sec. II, we introduce the model for the coupled Hubbard chains, 
and apply bosonization methods to obtain low-energy effective theory. For later convenience, we also summarize the results known for a clean system without randomness. In Sec. III, we derive the scaling equations for a disordered system at half-filling. Sec. IV and V are devoted to the discussions about the fixed point properties in the half-filling case. In particular, we discuss in detail how the competition between the Mott transition and the Anderson transition occurs when the random potentials are introduced. In Sec. VI, we further take into account the effects of random hopping between two bands. Conclusion is given in Sec. VII.

\section{MODEL AND BOSONIZATION METHOD: SUMMARY OF THE RESULTS FOR CLEAN SYSTEMS}

We first summarize the results for a clean system without random potentials, and then investigate the effects of disorder on the resulting fixed points. Our model Hamiltonian in the clean case is given by,

$$
\begin{aligned}
H= & -t \sum_{i, s, \alpha} c_{i s \alpha}^{\dagger} c_{i+1 s \alpha}+\text { h.c. }+U \sum_{i, \alpha} n_{i \uparrow \alpha} n_{i \downarrow \alpha} \\
& -t_{\perp} \sum_{i, s}\left[c_{i s 1}^{\dagger} c_{i s 2}+\text { h.c. }\right]
\end{aligned}
$$

where $c_{i s \alpha}\left(c_{i s \alpha}^{\dagger}\right)$ is an annihilation (creation) operator for electrons with spin $s=\uparrow \downarrow$ and chain-index $\alpha=1,2$ at a site $i$, and $n_{i s \alpha}=c_{i s \alpha}^{\dagger} c_{i s \alpha}$. The last term is the hopping term between two chains. The kinetic energy part is diagonalized in terms of a new basis,

$$
\begin{aligned}
& \psi_{s 1}(k)=\frac{c_{s 1}(k)-c_{s 2}(k)}{\sqrt{2}} \\
& \psi_{s 2}(k)=\frac{c_{s 1}(k)+c_{s 2}(k)}{\sqrt{2}} .
\end{aligned}
$$

The dispersion relations in this basis are given by $\varepsilon_{1 k}=$ $-2 t \cos k+t_{\perp}$ and $\varepsilon_{2 k}=-2 t \cos k-t_{\perp}$, resulting in the two decoupled bands. For $t_{\perp}>2 t$, there exists a band gap between these two bands. In the following we restrict our arguments to the interesting case of $t_{\perp}<2 t$, where the chemical potential may cross the two bands both at and near half-filling. We apply abelian bosonizatipn methods to this model to obtain the scaling equations.20121 In the presence of spin degrees of freedom, non-abelian bosonization is generally more convenient for preserving $\mathrm{SU}(2)$ symmetry.22 23 However, in our model the backward scattering due to impurities is expressed in terms of the operators which are not included in the operator content of SU(2) Wess-ZuminoWitten model. Therefore we exploit conventional abelian bosonization methods by carefully dealing with $\mathrm{SU}(2)$ symmetry of spin degrees of freedom. Passing to the continuum limit, we linearize the dispersions around the
Fermi momenta $k_{\mathrm{F} 1,2}=\left|\cos ^{-1}\left(\left( \pm t_{\perp}-\mu\right) / 2 t\right)\right|(\mu$ : chemical potential), and divide the electron operators into leftand right-going parts, $\psi_{s 1} \sim \exp \left(i \sqrt{4 \pi} \phi_{s 1 L}(x)+i k_{\mathrm{F} 1} x\right)+$ $\exp \left(-i \sqrt{4 \pi} \phi_{s 1 R}(x)-i k_{\mathrm{F} 1} x\right)$, etc. Defining the new boson phase fields,

$$
\begin{aligned}
& \phi_{+\rho}(x)=\frac{\phi_{\uparrow 1}(x)+\phi_{\downarrow 1}(x)+\phi_{\uparrow 2}(x)+\phi_{\downarrow 2}(x)}{2}, \\
& \phi_{-\rho}(x)=\frac{\phi_{\uparrow 1}(x)+\phi_{\downarrow 1}(x)-\phi_{\uparrow 2}(x)-\phi_{\downarrow 2}(x)}{2}, \\
& \phi_{+\sigma}(x)=\frac{\phi_{\uparrow 1}(x)-\phi_{\downarrow 1}(x)+\phi_{\uparrow 2}(x)-\phi_{\downarrow 2}(x)}{2}, \\
& \phi_{-\sigma}(x)=\frac{\phi_{\uparrow 1}(x)-\phi_{\downarrow 1}(x)-\phi_{\uparrow 2}(x)+\phi_{\downarrow 2}(x)}{2},
\end{aligned}
$$

with $\phi_{s 1(2)}(x) \equiv \phi_{s 1(2) L}(x)+\phi_{s 1(2) R}(x)(s=\uparrow, \downarrow)$, and their canonical conjugate momentum fields, $\Pi_{+\rho}(x)=$ $\partial_{x} \theta_{+\rho}(x) \equiv \partial_{x}\left(\phi_{+\rho L}-\phi_{+\rho R}\right)$, etc., we write down the low-energy effective Hamiltonian,

$$
\begin{aligned}
& H=H_{0}+H_{1}+H_{2}, \\
& H_{0}=\sum_{a= \pm, \nu=\rho, \sigma} \int d x\left[\frac{v_{a \nu}}{2 K_{a \nu}}\left(\partial_{x} \phi_{a \nu}(x)\right)^{2}\right. \\
& \left.+\frac{v_{a \nu} K_{a \nu}}{2} \Pi_{a \nu}^{2}(x)\right], \\
& H_{1}=U \int \frac{d x}{\alpha} \cos \left(\sqrt{8 \pi} \phi_{1 \rho}+\delta_{1} x\right) \\
& +U \int \frac{d x}{\alpha} \cos \left(\sqrt{8 \pi} \phi_{2 \rho}+\delta_{2} x\right), \\
& H_{2}=U_{1} \int \frac{d x}{\alpha} \cos \sqrt{4 \pi} \theta_{-\rho} \cos \sqrt{4 \pi} \phi_{-\sigma} \\
& +U_{2} \int \frac{d x}{\alpha} \cos \sqrt{4 \pi} \theta_{-\rho} \cos \sqrt{4 \pi} \phi_{+\sigma} \\
& +U_{3} \int \frac{d x}{\alpha} \cos \sqrt{4 \pi} \theta_{-\rho} \cos \left(\sqrt{4 \pi} \phi_{+\rho}+\delta x\right) \\
& +U_{4} \int \frac{d x}{\alpha} \cos \sqrt{4 \pi} \theta_{-\sigma} \cos \sqrt{4 \pi} \phi_{+\sigma} \\
& +U_{5} \int \frac{d x}{\alpha} \cos \sqrt{4 \pi} \theta_{-\sigma} \cos \left(\sqrt{4 \pi} \phi_{+\rho}+\delta x\right) \\
& +U_{6} \int \frac{d x}{\alpha} \cos \left(\sqrt{4 \pi} \phi_{+\rho}+\delta x\right) \cos \sqrt{4 \pi} \phi_{-\sigma} \\
& +U_{7} \int \frac{d x}{\alpha} \cos \sqrt{4 \pi} \phi_{-\sigma} \cos \sqrt{4 \pi} \phi_{+\sigma} \\
& +U_{8} \int \frac{d x}{\alpha} \cos \left(\sqrt{4 \pi} \phi_{+\rho}+\delta x\right) \cos \sqrt{4 \pi} \phi_{+\sigma} \\
& +U_{9} \int \frac{d x}{\alpha} \cos \sqrt{4 \pi} \theta_{-\sigma} \cos \sqrt{4 \pi} \theta_{-\rho},
\end{aligned}
$$

where $\delta_{1,2}=4 k_{\mathrm{F} 1,2}-2 \pi$, and $\delta=2\left(k_{\mathrm{F} 1}+k_{\mathrm{F} 2}\right)-2 \pi$. $H_{1}$ and $H_{2}$ are, respectively, the umklapp scattering terms within each band and the other scattering terms of electron-electron interaction. For on-site Coulomb interaction, initially $U_{i}=U$ for $i=1 \sim 7$ and $U_{8}=U_{9}=0$. We have dropped the terms which include an oscillating factor $\exp \left( \pm 2 i\left(k_{\mathrm{F} 1}-k_{\mathrm{F} 2}\right) x\right)$, because these terms are 
irrelevant in the long wave-length limit. We have also omitted the forward scattering terms of electron-electron interaction, which just renormalize the TL parameters.

We first consider the case of half-filling $\delta=0$. Then $H_{1}$-term is irrelevant and can be discarded after the renormalization procedure because of the oscillating factors. In general, the above Hamiltonian produces the mass gaps both for the $( \pm)$-charge sectors and $( \pm)$-spin sectors. In particular, it is to be noted that there are two possibilities for the gap formation of the (-)-spin sector; the spin gap is generated owing to locking of the $\phi_{-\sigma}$ or $\theta_{-\sigma}$ field. In this connection, we wish to mention that there is a special case for which some peculiar behavior in the mass generation is observed, i.e. the case of $U_{1}=U_{2}=U_{3}=U_{4}=U_{5}=U_{6}=U_{7}=U_{8}=U_{9}$ and $K_{-\sigma}=1$ where the model has special symmetry enhancement. 14 This situation realizes in the case with a long-range electron-electron interaction between parallel spins, $H^{\prime}=V \sum_{i, s=\uparrow \downarrow} n_{i s} n_{i+1 s}$, where the initial values of $U_{8}$ and $U_{9}$ are non-zero. To see the special symmetry, we rewrite the interaction term $\mathrm{H}_{2}$ as,

$$
\begin{aligned}
H_{2} & =U_{1} \int \frac{d x}{\alpha}\left[\left(\cos \sqrt{4 \pi} \phi_{+\rho}+\cos \sqrt{4 \pi} \phi_{+\sigma}\right.\right. \\
& \left.+\cos \sqrt{4 \pi} \phi_{-\rho}\right)\left(\cos \sqrt{4 \pi} \phi_{-\sigma}+\cos \sqrt{4 \pi} \theta_{-\sigma}\right) \\
& +\cos \sqrt{4 \pi} \theta_{-\rho} \cos \sqrt{4 \pi} \phi_{+\sigma} \\
& +\cos \sqrt{4 \pi} \theta_{-\rho} \cos \sqrt{4 \pi} \phi_{+\rho} \\
& \left.+\cos \sqrt{4 \pi} \phi_{+\sigma} \cos \sqrt{4 \pi} \phi_{+\rho}\right] .
\end{aligned}
$$

Then the Hamiltonian is invariant under the dual transformation $\phi_{-\sigma} \leftrightarrow \theta_{-\sigma}$. This is nothing but the KramersWannier symmetry of the Ising model. Thus only the half part of the boson degrees of freedom in the (-)-spin sector is massive, and the other half-part is decoupled and forms a massless mode of the Ising class with the central charge $c=1 / 2.14$ The microscopic model given by eq.(1) does not have this special symmetry, so that we can proceed with the following analysis by assuming that all the low-energy excitations are gapful.

We investigate the effects of disorder on these fixed points in the following sections. We note that the effects of quenched disorder on 1D interactipg electron systems have been extensively studied before.24 27 Our approach is a generalization of these previous studies. We introduce weak disorder potentials into the model (1),

$$
\begin{aligned}
H_{d i s}= & H_{f}+H_{b}, \\
H_{f}= & \int d x \sum_{s=\uparrow \downarrow} \eta_{1}(x)\left[\psi_{s 1 L}^{\dagger}(x) \psi_{s 1 L}(x)+\psi_{s 1 R}^{\dagger}(x) \psi_{s 1 R}(x)\right] \\
+ & \int d x \sum_{s=\uparrow \downarrow} \eta_{2}(x)\left[\psi_{s 2 L}^{\dagger}(x) \psi_{s 2 L}(x)+\psi_{s 2 R}^{\dagger}(x) \psi_{s 2 R}(x)\right] \\
+ & \int d x \sum_{s=\uparrow \downarrow} \eta_{3}(x)\left[e ^ { i ( k _ { \mathrm { F } 1 } - k _ { \mathrm { F } 2 } ) x } \left(\psi_{s 1 L}^{\dagger}(x) \psi_{s 2 L}(x)\right.\right. \\
& \left.\left.+\psi_{s 2 R}^{\dagger}(x) \psi_{s 1 R}(x)\right)+h . c .\right], \\
H_{b}= & \int d x \sum_{s=\uparrow \downarrow}\left[\xi_{1}(x) e^{2 i k_{\mathrm{F} 1}} \psi_{s 1 L}^{\dagger}(x) \psi_{s 1 R}(x)+h . c .\right] \\
& +\int d x \sum_{s=\uparrow \downarrow}\left[\xi_{2}(x) e^{2 i k_{\mathrm{F} 2}} \psi_{s 2 L}^{\dagger}(x) \psi_{s 2 R}(x)+h . c .\right] \\
& +\int d x \sum_{s=\uparrow \downarrow}\left[\xi _ { 3 } ( x ) e ^ { i ( k _ { \mathrm { F } 1 } + k _ { \mathrm { F } 2 } ) x } \left(\psi_{s 1 L}^{\dagger}(x) \psi_{s 2 R}(x)\right.\right. \\
& \left.\left.+\psi_{s 2 L}^{\dagger}(x) \psi_{s 1 R}(x)\right)+h . c .\right],
\end{aligned}
$$

where $H_{f}$ is the forward scattering part, and $H_{b}$ is the backward scattering part. $\eta_{i}(x)$ and $\xi_{i}(x)(i=1,2)$ are the random potential fields within each band for forward and backward scatterings, respectively. The $\eta_{3}(x)$ and $\xi_{3}(x)$ terms represent the random hopping between two splitted bands. We assume that these random fields obey the Gaussian distribution law, $\left\langle\eta_{i}(x) \eta_{j}\left(x^{\prime}\right)\right\rangle=$ $D_{\eta_{i}} \delta_{i j} \delta\left(x-x^{\prime}\right),\left\langle\xi_{i}(x) \xi_{j}^{*}\left(x^{\prime}\right)\right\rangle=D_{\xi_{i}} \delta_{i j} \delta\left(x-x^{\prime}\right)$, and $D_{\eta(\xi) 1}=D_{\eta(\xi) 2}$. We first consider the case of $D_{\eta 3}=$ $D_{\xi 3}=0$ in Sec. III, IV, and V, and take into account these random hopping terms in Sec. VI.

\section{SCALING EQUATIONS FOR DISORDERED SYSTEMS IN THE CASE WITHOUT RANDOM INTERBAND HOPPING}

In the absence of the $\eta_{3}$ and $\xi_{3}$ terms, we bosonize the random potential terms (14) and (15), and average over the random fields using the replica trick as in ref. 27. Then the random potential terms in the action are given by,

$$
\begin{aligned}
S_{d i s}= & -\frac{2 D_{\eta+}}{\pi} \int d x \int d \tau \int d \tau^{\prime} \sum_{i, j}^{n} \partial_{x} \phi_{+\rho}^{i}(x, \tau) \partial_{x} \phi_{+\rho}^{j}\left(x, \tau^{\prime}\right)-\frac{2 D_{\eta-}}{\pi} \int d x \int d \tau \int d \tau^{\prime} \sum_{i, j}^{n} \partial_{x} \phi_{-\rho}^{i}(x, \tau) \partial_{x} \phi_{-\rho}^{j}\left(x, \tau^{\prime}\right) \\
& -\frac{2 D_{A+}}{\pi} \int d x \int d \tau \int d \tau^{\prime} \sum_{i, j}^{n} \frac{1}{v_{+\rho}^{2}} \partial_{\tau} \phi_{+\rho}^{i}(x, \tau) \partial_{\tau} \phi_{+\rho}^{j}\left(x, \tau^{\prime}\right) \\
& -\frac{2 D_{A-}}{\pi} \int d x \int d \tau \int d \tau^{\prime} \sum_{i, j}^{n} \frac{1}{v_{-\rho}^{2}} \partial_{\tau} \phi_{-\rho}^{i}(x, \tau) \partial_{\tau} \phi_{-\rho}^{j}\left(x, \tau^{\prime}\right) \\
& -\frac{D_{\xi 1}}{\alpha^{2}} \int d x \int d \tau \int d \tau^{\prime} \sum_{i, j}^{n} \cos \sqrt{\pi}\left(\phi_{+\sigma}^{i}(x, \tau)+\phi_{-\sigma}^{i}(x, \tau)\right) \cos \sqrt{\pi}\left(\phi_{+\sigma}^{j}(x, \tau)+\phi_{-\sigma}^{j}(x, \tau)\right)
\end{aligned}
$$




$$
\begin{aligned}
& \times \cos \sqrt{\pi}\left(\phi_{+\rho}^{i}(x, \tau)+\phi_{-\rho}^{i}(x, \tau)-\phi_{+\rho}^{j}\left(x, \tau^{\prime}\right)-\phi_{-\rho}^{j}\left(x, \tau^{\prime}\right)\right) \\
& -\frac{D_{\xi 2}}{\alpha^{2}} \int d x \int d \tau \int d \tau^{\prime} \sum_{i, j}^{n} \cos \sqrt{\pi}\left(\phi_{+\sigma}^{i}(x, \tau)-\phi_{-\sigma}^{i}(x, \tau)\right) \cos \sqrt{\pi}\left(\phi_{+\sigma}^{j}(x, \tau)-\phi_{-\sigma}^{j}(x, \tau)\right) \\
& \times \cos \sqrt{\pi}\left(\phi_{+\rho}^{i}(x, \tau)-\phi_{-\rho}^{i}(x, \tau)-\phi_{+\rho}^{j}\left(x, \tau^{\prime}\right)+\phi_{-\rho}^{j}\left(x, \tau^{\prime}\right)\right),
\end{aligned}
$$

where $i, j$ are replica indices, and initially $D_{\eta \pm}=\left(D_{\eta 1}+\right.$ $\left.D_{\eta 2}\right) / 2$. The $D_{A \pm}$ terms are generated in the process of renormalization, though initially $D_{A \pm}=0.17$ The $D_{A \pm}$ term is equivalent to the interaction with random gauge fields,

$$
H_{r g \pm}=-\sqrt{\frac{2}{\pi}} \int d x A_{ \pm}(x) \partial_{x} \theta_{ \pm \rho}
$$

where the random gauge fields $A_{ \pm}(x)$ obey the Gaussian distribution law, $\left\langle A_{\alpha}(x) A_{\beta}\left(x^{\prime}\right)\right\rangle=D_{A \alpha} \delta_{\alpha \beta} \delta\left(x-x^{\prime}\right)$ $(\alpha, \beta= \pm)$. The random fields $\eta_{ \pm}(x)$ and $A_{ \pm}(x)$ can be incorporated into the shift of the phase fields.27.17 Defining the new fields,

$$
\begin{aligned}
\tilde{\phi}_{ \pm \rho}(x) & \equiv \phi_{ \pm \rho}(x)+\tilde{\eta}_{ \pm}(x) \\
\tilde{\eta}_{ \pm}(x) & \equiv \frac{K_{ \pm \rho}}{v_{ \pm \rho}} \sqrt{\frac{2}{\pi}} \int^{x} d x^{\prime} \eta_{ \pm}\left(x^{\prime}\right) \\
\tilde{\theta}_{ \pm \rho}(x) & \equiv \theta_{ \pm \rho}(x)+\tilde{A}_{ \pm}(x) \\
\tilde{A}_{ \pm}(x) & \equiv \frac{1}{K_{ \pm \rho} v_{ \pm \rho}} \sqrt{\frac{2}{\pi}} \int^{x} d x^{\prime} A_{ \pm}\left(x^{\prime}\right)
\end{aligned}
$$

where $\left\langle\eta_{\alpha}(x) \eta_{\beta}\left(x^{\prime}\right)\right\rangle=D_{\eta \alpha} \delta_{\alpha \beta} \delta\left(x-x^{\prime}\right)(\alpha, \beta= \pm)$, we can absorb the $\eta_{ \pm}(x)$ and $A_{ \pm}(x)$ terms into the Gaussian part of the Hamiltonian. Thus these terms do not affect the massless modes apart from a minor change in correlation functions.27. 17 However, as we will see momentarily, these terms are important for suppressing the charge and spin gaps in the massive sectors.

We now derive the scaling equations for the above effective Hamiltonian. Since we have so many coupling constants, it is pretty difficult to analyze all scaling equations even at one-loop level. We here ignore the renormalization of velocities for simplicity. In some previous studies11.15, it was shown that even with this approximation, the mass generation can be described rather well qualitatively for clean systems. This is partly because in the clean case the mass generation is mainly determined by the scaling dimensions of the relevant operators which are not affected by the renormalization of velocities in the lowest order. Thus this approximation gives a qualitatively correct answer for the mass generation in the clean case. We expect that this may be also the case so far as we are concerned with weak disorder. Using the operator product expansion of the $\mathrm{U}(1)$ Gaussian model, and taking the replica limit $n \rightarrow 0$, we have the scaling equations for dimensionless couplings defined by $\tilde{U}_{i}=U_{i} / v_{+\rho}, \tilde{D}_{\eta, A \pm}=D_{\eta, A \pm} / v_{ \pm \rho}^{2}$, and $\tilde{D}_{\xi i}=D_{\xi i} / v_{+\rho}^{2}$

$$
\begin{aligned}
& \frac{d \tilde{U}_{1}}{d l}=\left(2-\frac{1}{K_{-\rho}}-K_{-\sigma}\right) \tilde{U}_{1} \\
& -\frac{\tilde{U}_{3} \tilde{U}_{6}}{2}-\frac{\tilde{U}_{2} \tilde{U}_{7}}{2}-\frac{2 \tilde{D}_{A-} \tilde{U}_{1}}{\pi^{2} K_{-\rho}^{2}} \\
& \frac{d \tilde{U}_{2}}{d l}=\left(2-\frac{1}{K_{-\rho}}-K_{+\sigma}\right) \tilde{U}_{2} \\
& -\frac{\tilde{U}_{3} \tilde{U}_{8}}{2}-\frac{\tilde{U}_{1} \tilde{U}_{7}}{2}-\frac{2 \tilde{D}_{A-} \tilde{U}_{2}}{\pi^{2} K_{-\rho}^{2}}, \\
& \frac{d \tilde{U}_{3}}{d l}=\left(2-\frac{1}{K_{-\rho}}-K_{+\rho}\right) \tilde{U}_{3}-\frac{\tilde{U}_{1} \tilde{U}_{6}}{2}-\frac{\tilde{U}_{5} \tilde{U}_{9}}{2} \\
& -\frac{2 \tilde{D}_{A-} \tilde{U}_{3}}{\pi^{2} K_{-\rho}^{2}}-\frac{2 \tilde{D}_{\eta+} K_{+\rho}^{2} \tilde{U}_{3}}{\pi^{2}} \\
& \frac{d \tilde{U}_{4}}{d l}=\left(2-\frac{1}{K_{-\sigma}}-K_{+\sigma}\right) \tilde{U}_{4}-\frac{\tilde{U}_{2} \tilde{U}_{9}}{2}-\frac{\tilde{U}_{5} \tilde{U}_{8}}{2}, \\
& \frac{d \tilde{U}_{5}}{d l}=\left(2-\frac{1}{K_{-\sigma}}-K_{+\rho}\right) \tilde{U}_{5}-\frac{\tilde{U}_{4} \tilde{U}_{8}}{2}-\frac{\tilde{U}_{3} \tilde{U}_{9}}{2} \\
& -\frac{2 \tilde{D}_{\eta+} K_{+\rho}^{2} \tilde{U}_{5}}{\pi^{2}} \\
& \frac{d \tilde{U}_{6}}{d l}=\left(2-K_{-\sigma}-K_{+\rho}\right) \tilde{U}_{6}-\frac{\tilde{U}_{1} \tilde{U}_{3}}{2}-\frac{\tilde{U}_{7} \tilde{U}_{8}}{2} \\
& -\frac{2 \tilde{D}_{\eta+} K_{+\rho}^{2} \tilde{U}_{6}}{\pi^{2}} \\
& \frac{d \tilde{U}_{7}}{d l}=\left(2-K_{-\sigma}-K_{+\sigma}\right) \tilde{U}_{7}-\frac{\tilde{U}_{1} \tilde{U}_{2}}{2}-\frac{\tilde{U}_{6} \tilde{U}_{8}}{2} \\
& -\tilde{D}_{\xi 1}-\tilde{D}_{\xi 2} \\
& \frac{d \tilde{U}_{8}}{d l}=\left(2-K_{+\sigma}-K_{+\rho}\right) \tilde{U}_{8}-\frac{\tilde{U}_{4} \tilde{U}_{5}}{2}-\frac{\tilde{U}_{6} \tilde{U}_{7}}{2} \\
& -\frac{2 \tilde{D}_{\eta+} K_{+\rho}^{2} \tilde{U}_{8}}{\pi^{2}} \\
& \frac{d \tilde{U}_{9}}{d l}=\left(2-\frac{1}{K_{-\rho}}-\frac{1}{K_{-\sigma}}\right) \tilde{U}_{9}-\frac{\tilde{U}_{2} \tilde{U}_{4}}{2}-\frac{\tilde{U}_{3} \tilde{U}_{5}}{2} \\
& -\frac{2 \tilde{D}_{A-} \tilde{U}_{9}}{\pi^{2} K_{-\rho}^{2}} \\
& \frac{d K_{+\rho}}{d l}=-\frac{\pi K_{+\rho}^{2}}{2}\left[\tilde{U}_{3}^{2}+\tilde{U}_{5}^{2}+\tilde{U}_{6}^{2}+\tilde{U}_{8}^{2}\right] \\
& -\frac{\pi K_{+\rho}^{2}}{16}\left(\tilde{D}_{\xi 1}+\tilde{D}_{\xi 2}\right) \text {, } \\
& \frac{d K_{-\rho}}{d l}=\frac{\pi}{2}\left[\tilde{U}_{1}^{2}+\tilde{U}_{2}^{2}+\tilde{U}_{3}^{2}+\tilde{U}_{9}^{2}\right] \\
& -\frac{\pi K_{-\rho}^{2}}{16}\left(\tilde{D}_{\xi 1}+\tilde{D}_{\xi 2}\right)
\end{aligned}
$$




$$
\begin{aligned}
\frac{d K_{+\sigma}}{d l}= & -\frac{\pi K_{+\sigma}^{2}}{2}\left[\tilde{U}_{2}^{2}+\tilde{U}_{4}^{2}+\tilde{U}_{7}^{2}+U_{8}^{2}\right] \\
& -\frac{\pi K_{+\sigma}^{2}}{16}\left(\tilde{D}_{\xi 1}+\tilde{D}_{\xi 2}\right) \\
\frac{d K_{-\sigma}}{d l}= & \frac{\pi}{2}\left[\tilde{U}_{4}^{2}+\tilde{U}_{5}^{2}+\tilde{U}_{9}^{2}\right]-\frac{\pi K_{-\sigma}^{2}}{2}\left[\tilde{U}_{1}^{2}+\tilde{U}_{6}^{2}+\tilde{U}_{7}^{2}\right] \\
- & \frac{\pi K_{-\sigma}^{2}}{16}\left(\tilde{D}_{\xi 1}+\tilde{D}_{\xi 2}\right) \\
\frac{d \tilde{D}_{\eta \pm}}{d l}= & \tilde{D}_{\eta \pm}+\pi^{2}\left(\tilde{D}_{\xi 1}^{2}+\tilde{D}_{\xi 2}^{2}\right) \\
\frac{d \tilde{D}_{A \pm}}{d l}= & \tilde{D}_{A \pm}+\pi^{2}\left(\tilde{D}_{\xi 1}^{2}+\tilde{D}_{\xi 2}^{2}\right) \\
\frac{d \tilde{D}_{\xi i}}{d l}= & \left(3-\frac{K_{+\sigma}}{2}-\frac{K_{-\sigma}}{2}-\frac{K_{+\rho}}{2}-\frac{K_{-\rho}}{2}\right) \tilde{D}_{\xi i} \\
& -\tilde{U}_{7} \tilde{D}_{\xi i}, \quad i=1,2
\end{aligned}
$$

In the absence of disorder, $D_{\eta \pm}=D_{A \pm}=D_{\xi i}=0$, the excitation gaps open above the ground state energy in the $( \pm)$-charge and $( \pm)$-spin sectors. We have two possibilities in the massive phase of the (-)-spin sector as mentioned in Sec. II. If $\tilde{U}_{1}, \tilde{U}_{6}$, and $\tilde{U}_{7}$ scale to strongcoupling regime, and the $\tilde{U}_{4}, \tilde{U}_{5}$, and $\tilde{U}_{9}$ terms are irrelevant, the phase field $\phi_{-\sigma}$ is locked, and the correlations for the operator $\exp \left(i a \theta_{-\sigma}\right)$ shows exponential decay. We refer to this case as case $A$. To the contrary, if the $\tilde{U}_{4}, \tilde{U}_{5}$, and $\tilde{U}_{9}$ terms are relevant, and the $\tilde{U}_{1}, \tilde{U}_{6}$, and $\tilde{U}_{7}$ terms are irrelevant, $\theta_{-\sigma}$ is locked. We refer to this case as case $B$. In the subsequent sections, we discuss the effects of disorder on these two kinds of the fixed points.

\section{DISORDERED FIXED POINT: $C A S E A$}

Here we consider the effects of quenched disorder on the fixed point of case $A$, where the phase field $\phi_{-\sigma}$ is locked, and $\exp \left(i a \theta_{-\sigma}\right)$ is the disorder parameter. In this case, the $\tilde{U}_{1}, \tilde{U}_{2}, \tilde{U}_{3}, \tilde{U}_{6}, \tilde{U}_{7}$, and $\tilde{U}_{8}$ terms are relevant, and the $\tilde{U}_{4}, \tilde{U}_{5}, \tilde{U}_{9}$ terms are irrelevant in the clean system. The sufficiently strong forward scattering due to impurities $\tilde{D}_{\eta+}$ may suppress the mass-generating interactions, $\tilde{U}_{3}, \tilde{U}_{6}$, and $\tilde{U}_{8}$, as seen from Eqs.(24), (27), and (29). It turns out from Eqs.(22) and (23) that this is not the case for the $\tilde{U}_{1}$ and $\tilde{U}_{2}$ terms which bear the charge gap in the (-)-sector, because they are not coupled with the random forward scattering. We note, however, that these terms should be suppressed in the presence of sufficiently strong random gauge fields $A_{-}$generated by random backward scattering due to impurities. Therefore in order to close the charge gap in the (-)-sector, one needs the random backward scattering due to impurities. This point makes a clear contrast to the case of a single chain model where the Mott-Hubbard gap is collapsed pnly by the random forward scattering due to impurities. 17

Note that the $\tilde{U}_{7}$ term which generates the spin gap in $\phi_{ \pm \sigma}$ fields is not affected by the random forward scatter- ing, because the random forward scattering couples only with the charge degrees of freedom. On the other hand, it is seen from Eq.(28) that the backward scattering due to impurities $\tilde{D}_{\xi i}$ may drive $\tilde{U}_{7}$ to a large negative value. However, if the initial values of $\tilde{D}_{\xi i}$ are small enough compared to that of $\tilde{U}_{7}$, the spin gaps of $\phi_{ \pm \sigma}$ fields generated by the $\tilde{U}_{7}$ term may survive. Based on this observation, we start with the limiting case $\tilde{U}_{7} \rightarrow+\infty$ for simplicity. Then after the spin gap formation, we can eliminate the spin degrees of freedom from the scaling equations. Suppressing irrelevant terms, we have the reduced scaling equations,

$$
\begin{aligned}
\frac{d \tilde{U}_{1}}{d l}= & \left(2-\frac{1}{K_{-\rho}}\right) \tilde{U}_{1}-\frac{\tilde{U}_{3} \tilde{U}_{6}}{2}-\frac{2 \tilde{D}_{A-} \tilde{U}_{1}}{\pi^{2} K_{-\rho}^{2}}, \\
\frac{d \tilde{U}_{2}}{d l}= & \left(2-\frac{1}{K_{-\rho}}\right) \tilde{U}_{2}-\frac{\tilde{U}_{3} \tilde{U}_{8}}{2}-\frac{2 \tilde{D}_{A-} \tilde{U}_{2}}{\pi^{2} K_{-\rho}^{2}}, \\
\frac{d \tilde{U}_{3}}{d l}= & \left(2-\frac{1}{K_{-\rho}}-K_{+\rho}\right) \tilde{U}_{3}-\frac{\tilde{U}_{1} \tilde{U}_{6}}{2}-\frac{\tilde{U}_{5} \tilde{U}_{9}}{2} \\
& -\frac{2 \tilde{D}_{A-} \tilde{U}_{3}}{\pi^{2} K_{-\rho}^{2}}-\frac{2 \tilde{D}_{\eta+} K_{+\rho}^{2} \tilde{U}_{3}}{\pi^{2}}, \\
\frac{d \tilde{U}_{6}}{d l}= & \left(2-K_{+\rho}\right) \tilde{U}_{6}-\frac{\tilde{U}_{1} \tilde{U}_{3}}{2}-\frac{2 \tilde{D}_{\eta+} K_{+\rho}^{2} \tilde{U}_{6}}{\pi^{2}}, \\
\frac{d \tilde{U}_{8}}{d l}= & \left(2-K_{+\rho}\right) \tilde{U}_{8}-\frac{2 \tilde{D}_{\eta+} K_{+\rho}^{2} \tilde{U}_{8}}{\pi^{2}}, \\
\frac{d K_{+\rho}}{d l}= & -\frac{\pi K_{+\rho}^{2}}{2}\left[\tilde{U}_{3}^{2}+\tilde{U}_{6}^{2}+\tilde{U}_{8}^{2}\right] \\
& -\frac{\pi K_{+\rho}^{2}}{16}\left(\tilde{D}_{\xi 1}+\tilde{D}_{\xi 2}\right), \\
\frac{d K_{-\rho}}{d l}= & \frac{\pi}{2}\left[\tilde{U}_{1}^{2}+\tilde{U}_{2}^{2}+\tilde{U}_{3}^{2}\right]-\frac{\pi K_{-\rho}^{2}}{16}\left(\tilde{D}_{\xi 1}+\tilde{D}_{\xi 2}\right), \\
\frac{d \tilde{D}_{\xi i}}{d l}= & \left(3-\frac{K_{+\rho}}{2}-\frac{K_{-\rho}}{2}\right) \tilde{D}_{\xi i}, \quad i=1,2 .
\end{aligned}
$$

Since initially $\tilde{D}_{\eta \pm}>\tilde{D}_{A \pm}=0$, and they obey the same scaling equations (35) and (36), $\tilde{D}_{\eta \pm}$ flows into strongcoupling regime faster than $\tilde{D}_{A \pm}$. Thus there exists a parameter region where the $\tilde{U}_{3}, \tilde{U}_{6}$, and $\tilde{U}_{8}$ terms are suppressed for sufficiently large $\tilde{D}_{\eta+}$, though the $\tilde{U}_{1}$ and $\tilde{U}_{2}$ terms are still relevant. Then the charge gap in (+)charge mode is collapsed. Since the $\theta_{-\rho}$ field is locked due to the $\tilde{U}_{1}$ and $\tilde{U}_{2}$ terms, $K_{-\rho}$ scales to a large value, and the $\tilde{D}_{\xi i}$ terms are suppressed, as seen from Eq.(45). However, these terms are proved to be relevant, after one integrates out the $\phi_{-\rho}$ fifild, following the argument by Orignac and Giamarchi16. as in the case away from halfflling 16 Then the $(+)$-charge mode is in the Anderson localized state, while the (-)-charge mode is still in the Mott insulating state.

As the value of $\tilde{D}_{A-}$ becomes larger, the $\tilde{U}_{1}, \tilde{U}_{2}$ terms are suppressed, and all excitation gaps are collapsed. Then, the backward scattering due to impurities, $\tilde{D}_{\xi i}$ is relevant, and consequently the Anderson localization 
occurs. $\tilde{U}_{7}$ flows to a large negative value because of the $\tilde{D}_{\xi i}$ term, as seen from Eq.(28). Thus the spin degrees of freedom are frozen. The fixed point is identified with the pinned CDW state which is similar to that found in the disordered single chain model 27

In order to confirm the above arguments, we solve the scaling equations (22)-(37) numerically for several initial values of coupling constants. The numerical results for the flow of coupling constants are shown in FIG.1. In order to realize the massive spin state due to the locking of $\phi_{-\sigma}$ in the clean system, we have chosen the initial value of the TL liquid parameter in the $(-)$-spin sector as $K_{-\sigma}(0)=0.86$. The other TL parameters are set to be $K_{ \pm \rho}(0)=0.98$ and $K_{+\sigma}(0)=1.0$. For these initial values, the system definitely flows toward the low-energy fixed point of case $A$ in the absence of disorder. In FIG.1(a) the results in the case without quenched disorder are shown. $\tilde{U}_{1}, \tilde{U}_{2}, \tilde{U}_{3}, \tilde{U}_{6}, \tilde{U}_{7}$, and $\tilde{U}_{8}$ flow into a strong-coupling regime, while $\tilde{U}_{4}, \tilde{U}_{5}$, and $\tilde{U}_{9}$ scale to 0 . As a consequence, the phase fields $\phi_{+\rho}$, $\theta_{-\rho} . \phi_{ \pm \sigma}$ are locked. This fixed point is stable against the disorder weaker than mass-generating interactions, because of the presence of excitation gaps in all modes. As the quenched disorder becomes stronger, some different fixed points manifest themselves. In FIG.1(b), we show the results for the initial values $\tilde{D}_{\eta \pm}(0)=0.9$ and $\tilde{D}_{\xi i}(0)=0.005$. Although $\tilde{U}_{1}, \tilde{U}_{2}$ and $\tilde{U}_{7}$ still scale to a strong-coupling regime, $\tilde{U}_{3}, \tilde{U}_{6}$, and $\tilde{U}_{8}$ scale to 0 . As a result, the charge gap in the $(+)$-mode is collapsed. Since $\tilde{D}_{\xi i}$ is relevant, the charge mode in the $(+)$-sector is localized, though the charge mode in the $(-)$-sector is still massive. Thus the Anderson localized state and the Mott insulating state coexist in this phase. Since $\tilde{U}_{7} \rightarrow-\infty$, the spin degrees of freedom are frozen, and may be in the random spin singlet state. In FIG.1(c), the results for $D_{\eta \pm}(0)=0.9$ and $D_{\xi i}(0)=0.01$ are shown. In this case, all mass-generating interactions except $\tilde{U}_{7}$ in Eq.(11) flow to 0 . The charge gaps are thus collapsed in this phase. Since $\tilde{D}_{\xi i}$ scales to a strong-coupling regime, the Anderson localized state realizes. The relevance of the $\tilde{U}_{7}$ term implies freezing of spin degrees of freedom in the $( \pm)$-sectors.

In summary, we have found three kinds of fixed points in the presence of quenched disorder: (i) the clean fixed point where the disorder effect is entirely irrelevant, (ii) Anderson-insulator (I) where the (+)-charge mode is in the Anderson localized state, the (-)-charge mode is in the Mott insulating state, and the spin degrees of freedom are massive or frozen, (iii) Anderson-insulator (II) where all charge modes are in the Anderson localized state, and spin degrees of freedom are frozen, i.e. pinned CDW. We thus end up with the schematic phase diagram for charge degrees of freedom as shown in FIG.2.

\section{DISORDERED FIXED POINT: $C A S E B$}

Here we consider the effects of disorder on the fixed point of case $B: \theta_{-\sigma}$ is locked, and $\exp \left(i a \phi_{-\sigma}\right)$ is the disorder parameter field in the absence of quenched disorder. In this case, the $\tilde{U}_{2}, \tilde{U}_{3}, \tilde{U}_{4}, \tilde{U}_{5}, \tilde{U}_{8}$, and $\tilde{U}_{9}$ terms are relevant in the clean system. We see from Eq.(34) that $K_{-\sigma}$ is decreased by the backward scattering due to impurities $\tilde{D}_{\xi i}$. Thus it suppresses the growth of $\tilde{U}_{4}$, $\tilde{U}_{5}$, and $\tilde{U}_{9}$ and prevents the spin gap formation due to locking of the $\theta_{-\sigma}$ field. Moreover it follows from Eq.(28) that if the $\tilde{D}_{\xi i}$ term is relevant, $\tilde{U}_{7}$ flows to a large negative value, resulting in freezing of the spin degrees of freedom, and the suppression of the $\tilde{U}_{4}$ term as in Sec. IV. However for sufficiently small $\tilde{D}_{\xi i}$, the $\tilde{U}_{4}$ term is relevant and the spin gap opens owing to locking of $\theta_{-\sigma}$. After the generation of the spin gap due to locking of the $\theta_{-\sigma}$ and $\phi_{+\sigma}$ fields, we can eliminate the spin degrees of freedom, and obtain the scaling equations for the charge degrees of freedom,

$$
\begin{aligned}
\frac{d \tilde{U}_{2}}{d l}= & \left(2-\frac{1}{K_{-\rho}}\right) \tilde{U}_{2}-\frac{\tilde{U}_{3} \tilde{U}_{8}}{2}-\frac{2 \tilde{D}_{A-} \tilde{U}_{2}}{\pi^{2} K_{-\rho}^{2}}, \\
\frac{d \tilde{U}_{3}}{d l}= & \left(2-\frac{1}{K_{-\rho}}-K_{+\rho}\right) \tilde{U}_{3}-\frac{\tilde{U}_{5} \tilde{U}_{9}}{2} \\
& -\frac{2 \tilde{D}_{A-} \tilde{U}_{3}}{\pi^{2} K_{-\rho}^{2}}-\frac{2 \tilde{D}_{\eta+} K_{+\rho}^{2} \tilde{U}_{3}}{\pi^{2}}, \\
\frac{d \tilde{U}_{5}}{d l}= & \left(2-K_{+\rho}\right) \tilde{U}_{5}-\frac{\tilde{U}_{3} \tilde{U}_{9}}{2} \\
& -\frac{2 \tilde{D}_{\eta+} K_{+\rho}^{2} \tilde{U}_{5}}{\pi^{2}}, \\
\frac{d \tilde{U}_{8}}{d l}= & \left(2-K_{+\rho}\right) \tilde{U}_{8}-\frac{2 \tilde{D}_{\eta+} K_{+\rho}^{2} \tilde{U}_{8}}{\pi^{2}}, \\
\frac{d \tilde{U}_{9}}{d l}= & \left(2-\frac{1}{K_{-\rho}}\right) \tilde{U}_{9}-\frac{\tilde{U}_{3} \tilde{U}_{5}}{2}-\frac{2 \tilde{D}_{A-} \tilde{U}_{9}}{\pi^{2} K_{-\rho}^{2}}, \\
\frac{d K_{+\rho}}{d l}= & -\frac{\pi K_{+\rho}^{2}}{2}\left[\tilde{U}_{3}^{2}+\tilde{U}_{5}^{2}+\tilde{U}_{8}^{2}\right], \\
& -\frac{\pi K_{+\rho}^{2}}{16}\left(\tilde{D}_{\xi 1}+\tilde{D}_{\xi 2}\right), \\
\frac{d K_{-\rho}}{d l}= & \frac{\pi}{2}\left[\tilde{U}_{2}^{2}+\tilde{U}_{3}^{2}+\tilde{U}_{9}^{2}\right] . \\
& -\frac{\pi K_{-\rho}^{2}}{16}\left(\tilde{D}_{\xi 1}+\tilde{D}_{\xi 2}\right), \\
\frac{d \tilde{D}_{\xi 1,2}}{d l}= & \left(3-2 K_{+\rho}-2 K_{-\rho}\right) \tilde{D}_{\xi i} .
\end{aligned}
$$

As pointed out in Sec. IV, $\tilde{D}_{\eta \pm}$ grows faster than $\tilde{D}_{A \pm}$. Thus there may exist a parameter region where $\tilde{D}_{\eta+}$ suppresses $\tilde{U}_{3}, \tilde{U}_{5}$, and $\tilde{U}_{8}$, but the $\tilde{U}_{2}$ and $\tilde{U}_{9}$ terms are still relevant, bearing the mass gap in the (-)-charge mode. In this parameter region, only the charge gap in the $(+)-$ charge sector is collapsed. The low-energy properties of 
this sector is determined by the $\tilde{D}_{\xi i}$-terms. After integrating out the massive (-)-charge mode 16 , we find that the scaling equation for the $\tilde{D}_{\xi 1,2}$-terms are changed to,

$$
\frac{d \tilde{D}_{\xi 1,2}}{d l}=\left(3-8 K_{+\rho}\right) \tilde{D}_{\xi i} .
$$

Thus the impurity backward scattering is suppressed in comparison with single chain systems. For $K_{+\rho}>3 / 8$, the $D_{\xi 1,2}$-terms are irrelevant. However it does not directly mean the occurrence of delocalization, since we should actually include the random hopping between two bands, $D_{\eta 3}, D_{\xi 3}$. In the case of $D_{\eta 3} \neq 0$ and $D_{\xi 3} \neq 0$, the CDW order couples directly to the $D_{\xi 3}$ term, and then the pinning of the CDW occurs for infinitesimally small $D_{\xi 3}$ as will be discussed in Sec. VI. Therefore the $(+)$-charge mode is in the Anderson localized state with the vanishing Drude weight and the non-zero charge susceptibility. For a sufficiently large value of $\tilde{D}_{A-}$, the charge gap in the (-)-mode may be also collapsed. All the charge modes are in the Anderson localized state.

In order to specify the low-energy fixed points correctly, we have solved the full scaling equations (22)-(37) numerically for some initial values of couplings. The numerical results are shown in FIG.3. In FIG.3(a), we display the results for clean systems. In order to realize the spin gapped state due to locking of $\theta_{-\sigma}$, we choose the initial values of the parameters $K_{-\sigma}(0)=1.1$, $K_{+\sigma}(0)=1.0$, and $K_{ \pm \rho}(0)=0.98$. For these parameters, $\tilde{U}_{2}, \tilde{U}_{3}, \tilde{U}_{4}, \tilde{U}_{5}, \tilde{U}_{8}$, and $\tilde{U}_{9}$ scale to a strong-coupling regime, and $\tilde{U}_{1}, \tilde{U}_{6}$, and $\tilde{U}_{7}$ scale to 0 . Then the phase fields $\phi_{+\rho}, \theta_{-\rho}, \phi_{+\sigma}$, and $\theta_{-\sigma}$ are locked. This fixed point with the spin and charge gaps is stable as far as disorder is much weaker than mass-generating interactions. In FIG.3(b), the results for $\tilde{D}_{\eta \pm}(0)=0.9$, and $\tilde{D}_{\xi i}(0)=0.007$ are shown. This flow ends up at the same fixed point as that shown in FIG.1(b), where the backward scattering due to impurities is relevant, and thus the Anderson localization occurs in the (+)-charge mode, though the (-)-charge mode is in the Mott insulating state. The spin degrees of freedom are also massive or frozen. Note that the freezing of the spin degrees of freedom in the (-)-sector is not due to locking of $\theta_{-\sigma}$ but due to $\phi_{-\sigma}$. Thus the fixed point properties of the $(-)$-spin sector are different from those for the clean case where locking of $\theta_{-\sigma}$ occurs. In FIG.3(c), we show the results for $\tilde{D}_{\eta \pm}(0)=0.9$ and $\tilde{D}_{\xi i}(0)=0.01$. In this case, the flows of coupling constants are qualitatively the same as those shown in FIG.1(c); i.e. all excitation gaps are collapsed, and the Anderson localized state with frozen spin degrees of freedom is realized. Thus for sufficiently strong impurity scattering, the disordered fixed point of case $B$ flows into the same class as that of case $A$. In summary, we have found three kinds of fixed points as in case $A$. The schematic phase diagram for the charge degrees of freedom is also given by FIG.2.

\section{EFFECTS OF RANDOM HOPPING BETWEEN TWO BANDS}

We now take into account the effects of random hopping between two bands, $\eta_{3}$ and $\xi_{3}$. First we consider only random forward scattering, and omit random backward scattering for a while. Going back to the fermion representation, we write down the kinetic energy part and the impurity forward scattering part of the Hamiltonian,

$$
\begin{aligned}
H_{0}^{\prime}= & \int d x \sum_{s=\uparrow \downarrow}\left[v_{1}\left(\psi_{s 1 L}^{\dagger} \partial_{x} \psi_{s 1 L}-\psi_{s 1 R}^{\dagger} \partial_{x} \psi_{s 1 R}\right)\right. \\
+ & \left.v_{2}\left(\psi_{s 2 L}^{\dagger} \partial_{x} \psi_{s 2 L}-\psi_{s 2 R}^{\dagger} \partial_{x} \psi_{s 2 R}\right)\right] \\
+ & \int d x \sum_{s=\uparrow \downarrow} \eta_{1}(x)\left[\psi_{s 1 L}^{\dagger}(x) \psi_{s 1 L}(x)+\psi_{s 1 R}^{\dagger}(x) \psi_{s 1 R}(x)\right] \\
+ & \int d x \sum_{s=\uparrow \downarrow} \eta_{2}(x)\left[\psi_{s 2 L}^{\dagger}(x) \psi_{s 2 L}(x)+\psi_{s 2 R}^{\dagger}(x) \psi_{s 2 R}(x)\right] \\
& +\int d x \sum_{s=\uparrow \downarrow} \eta_{3}(x)\left[e ^ { i ( k _ { \mathrm { F } 1 } - k _ { \mathrm { F } 2 } ) x } \left(\psi_{s 1 L}^{\dagger}(x) \psi_{s 2 L}(x)\right.\right. \\
& \left.\left.+\psi_{s 2 R}^{\dagger}(x) \psi_{s 1 R}(x)\right)+h . c .\right] .
\end{aligned}
$$

In the case of half-filling, $v_{1}=v_{2} \equiv v$ holds. Defining the spinor fields,

$$
\psi_{s L(R)}=\left(\begin{array}{c}
\psi_{s 1 L(R)} \\
\psi_{s 2 L(R)}
\end{array}\right),
$$

we cast the Hamiltonian Eq.(55) into the following form,

$$
\begin{aligned}
H_{0}^{\prime} & =\int d x \sum_{s=\uparrow \downarrow} v\left(\psi_{s L}^{\dagger} \partial_{x} \psi_{s L}-\psi_{s R}^{\dagger} \partial_{x} \psi_{s R}\right) \\
& +\int d x \sum_{s=\uparrow \downarrow} \psi_{s L}^{\dagger}\left(\frac{\eta_{1}+\eta_{2}}{2} \mathbf{1}+\frac{\eta_{1}-\eta_{2}}{2} \tau^{z}\right. \\
& \left.+\eta_{3} e^{i\left(k_{\mathrm{F} 1}-k_{\mathrm{F} 2}\right) x} \frac{\tau^{+}}{2}+\eta_{3}^{*} e^{-i\left(k_{\mathrm{F} 1}-k_{\mathrm{F} 2}\right) x} \frac{\tau^{-}}{2}\right) \psi_{s L} \\
& +\int d x \sum_{s=\uparrow \downarrow} \psi_{s R}^{\dagger}\left(\frac{\eta_{1}+\eta_{2}}{2} \mathbf{1}+\frac{\eta_{1}-\eta_{2}}{2} \tau^{z}\right. \\
& \left.+\eta_{3}^{*} e^{-i\left(k_{\mathrm{F} 1}-k_{\mathrm{F} 2}\right) x} \frac{\tau^{+}}{2}+\eta_{3} e^{i\left(k_{\mathrm{F} 1}-k_{\mathrm{F} 2}\right) x} \frac{\tau^{-}}{2}\right) \psi_{s R},
\end{aligned}
$$

where 1 is a $2 \times 2$ unit matrix, and $\tau^{z}, \tau^{ \pm}$are the Pauli matrices. This Hamiltonian can be diagonalized by using the unitary transformations defined by,

$$
\begin{aligned}
\tilde{\psi}_{s L}= & U_{L} \psi_{s L} \\
U_{L} \equiv & \exp \left(-\frac{i}{v} \int_{-\infty}^{x} d x^{\prime}\left[\frac{\eta_{1}\left(x^{\prime}\right)+\eta_{2}\left(x^{\prime}\right)}{2} \mathbf{1}\right.\right. \\
& +\frac{\eta_{1}\left(x^{\prime}\right)-\eta_{2}\left(x^{\prime}\right)}{2} \tau^{z} \\
& +\eta_{3}\left(x^{\prime}\right) e^{i\left(k_{\mathrm{F} 1}-k_{\mathrm{F} 2}\right) x^{\prime}} \frac{\tau^{+}}{2} \\
& \left.\left.+\eta_{3}^{*}\left(x^{\prime}\right) e^{-i\left(k_{\mathrm{F} 1}-k_{\mathrm{F} 2}\right) x^{\prime}} \frac{\tau^{-}}{2}\right]\right)
\end{aligned}
$$


and $U_{R}=U_{L}^{\dagger}$. We thus end up with the diagonalized form of $H_{0}^{\prime}$,

$$
H_{0}^{\prime}=\int d x \sum_{s} v\left(\tilde{\psi}_{s L}^{\dagger} \partial_{x} \tilde{\psi}_{s L}-\tilde{\psi}_{s R}^{\dagger} \partial_{x} \tilde{\psi}_{s R}\right)
$$

Therefore the forward scattering part of the random hopping can be incorporated into the free part of the Hamiltonian. Under the unitary transformation (59), the forward scattering terms of electron-electron interaction are invariant. The backward and umklapp scattering terms of electron-electron interaction (11) which may generate the mass gaps are multiplied with exponentially decaying factors by this transformation. Thus for sufficiently strong impurity forward scattering both of the charge and spin gaps collapse as in the previous sections.

Let us now take into account random backward scattering. The backward scattering term of random hopping is expressed as

$$
H_{\xi 3}=\int d x\left[\xi_{3}(x) e^{i\left(k_{\mathrm{F} 1}+k_{\mathrm{F} 2}\right) x} O_{C D W}(x)+\text { h.c. }\right] .
$$

The scaling equation for $D_{\xi 3}$ is given by

$$
\begin{aligned}
\frac{d \tilde{D}_{\xi 3}}{d l}= & \left(3-\frac{K_{+\rho}}{2}-\frac{1}{2 K_{-\rho}}-\frac{K_{+\sigma}}{2}-\frac{1}{2 K_{-\sigma}}\right. \\
& \left.-\tilde{U}_{4}\right) \tilde{D}_{\xi 3},
\end{aligned}
$$

where $\tilde{D}_{\xi 3}=D_{\xi 3} / v_{+\rho}^{2}$. It is seen that the $D_{\xi 3}$ term couples with the $U_{4}$ term. Then the scaling equation (25) is altered to

$$
\begin{aligned}
\frac{d \tilde{U}_{4}}{d l}= & \left(2-K_{+\sigma}-\frac{1}{K_{-\sigma}}\right) \tilde{U}_{4}-\tilde{U}_{2} \tilde{U}_{9}-\tilde{U}_{5} \tilde{U}_{8} \\
& -\tilde{D}_{\xi 3} .
\end{aligned}
$$

Therefore in the case that the mass gaps open due to locking of the $\phi_{+\rho}, \theta_{-\rho}, \phi_{+\sigma}$, and $\theta_{-\sigma}$ fields in the clean system (case $B), 3-K_{+\rho} / 2-1 / 2 K_{-\rho}-K_{+\sigma} / 2-1 / 2 K_{-\sigma}-$ $\tilde{U}_{4}>0$ holds, and the $D_{\xi 3}$ term is relevant. Then, the Anderson localization inevitably occurs in the $(+)$-charge sector provided that the impurity forward scattering suppresses the charge gap as mentioned in Sec. V. This fixed point is the pinned CDW. If the random backward scattering $\tilde{D}_{\xi 1,2}$ drives $\tilde{U}_{7}$ to $-\infty$, the $\phi_{-\sigma}$ field is locked. Then in the presence of the gap in the $(-)$-charge sector, integrating out the $\theta_{-\rho}$ field as in ref. 16, we find that the scaling equation 62 is changed to,

$$
\frac{d \tilde{D}_{\xi 3}}{d l}=\left(3-2 K_{+\rho}\right) \tilde{D}_{\xi 3} .
$$

Thus although the $\tilde{D}_{\xi 3}$-term is suppressed, it is still relevant for the repulsive interaction $K_{+\rho}<1$, and the Anderson localization occurs in the $(+)$-charge sector.

If the spin gap opens owing to locking of the $\phi_{-\sigma}$ field in the clean system (case $A$ ), the scaling equation for $\tilde{D}_{\xi 3}$ is also given by Eq.(64). Then the random hopping between two bands enhances the Anderson localization obtained in Sec. IV.

\section{CONCLUSION}

We have systematically studied the effects of quenched disorder on the spin-gapped state of two chain coupled Hubbard ladder model at half-filling. We have shown that a sufficiently strong forward scattering due to impurities and the random gauge fields generated by impurity backward scattering collapse all the charge gaps as well as the spin gaps, and consequently the Anderson localization takes place in all the charge sectors owing to the impurity backward scattering. We have also found the phase that one charge mode is in the Anderson localized state, while the other one is in the massive state.

This work was partly supported by a Grant-in-Aid from the Ministry of Education, Science and Culture.

${ }^{1}$ E. Dagotto, J. Riera. and D. J. Scalapino, Phys. Rev. B45, 5744 (1992).

${ }^{2}$ T. M. Rice, S. Gopalan, and M. Sigrist, Europhys. Lett. 23, 445 (1993); Phys. Rev. B49, 8901 (1994).

${ }^{3}$ R. M. Noack, S. White, D. J. Scalapino, Phys. Rev. Lett. 73, 882 (1994).

${ }^{4}$ M. Takano, Z. Hiroi, M. Azuma, and Y. Bando, J. Solid State Chem. 95, 230 (1991).

${ }^{5}$ R. S. Eccleston, T. Barnes, J. Brody, and J. W. Johnson, Phys. Rev. Lett. 73, 2626 (1994).

${ }^{6}$ M. Uehara, T. Nagata, J. Akimitsu, H. Takahashi, T. Mori, and K. Kinosita, J. Phys. Soc. Jpn. 65, 2764 (1996).

${ }^{7}$ A. M. Finkel'stein and A. I. Larkin, Phys. Rev. B47, 10461 (1993).

${ }^{8}$ M. Fabrizio, Phys. Rev. B48, 15838 (1993); M. Fabrizio and A. Parola, Phys. Rev. Lett. 70, 226 (1993).

${ }^{9}$ M. Imada, Phys. Rev. B48, 550 (1993).

${ }^{10}$ H. Tsunetsugu, M. Troyer, and T. M. Rice, Phys. Rev. B49, 16078 (1994).

${ }^{11}$ D. M. Khveshchenko and T. M. Rice, Phys. Rev. B50, 252 (1994); D. M. Khveshchenko, Phys. Rev. B50, 380 (1994).

12 S. Fujimoto and N. Kawakami, Phys. Rev. B52, 6189 (1995).

13 N. Nagaosa and M. Oshikawa, J. Phys. Soc. Jpn. 65, 2241 (1996).

14 H. J. Schulz, Phys. Rev. B53, 2959 (1996).

${ }^{15}$ L. Balents and M. P. A. Fisher, Phys. Rev. B53, 12133 (1996).

${ }^{16}$ E. Orignac and T. Giamarchi, Phys. Rev. B53, 10453 (1996).

17 S. Fujimoto and N. Kawakami, Phys. Rev. B 54, 11018 (1996). 
${ }^{18}$ M. Mori and H. Fukuyama, J. Phys. Soc. Jpn. 65, 3604 (1996).

${ }^{19}$ M. Yamanaka and M. Kohmoto, unpublished.

${ }^{20}$ V. J. Emery, in Highly Conducting One-Dimensional Solids, edited by J. T. Devreese, R. P. Evrard, and V. E. van Doren (Plenum, New York, 1979).

${ }^{21}$ F. D. M. Haldane, J. Phys. C 14, 2585 (1981).

${ }^{22}$ E. Witten, Comm. Math. Phys. 92, 455 (1984).

${ }^{23}$ I. Affleck, Nucl. Phys. B265, 409 (1986); in Fields, Strings and Critical Phenomena, ed. E. Brezin and J. Zinn-Justin (North-Holland, Amsterdam, 1990).

${ }^{24}$ S. T. Chui and J. W. Bray, Phys. Rev. B16, 1329 (1977)

${ }^{25}$ W. Appel, J. Phys. C 15, 1973 (1982); W. Appel and T. M. Rice, Phys. Rev. B26, 7063 (1982).

${ }^{26}$ Y. Suzumura and H. Fukuyama, J. Phys. Soc. Jpn. 52, 2870 (1983); 53, 3918 (1984).

27 T. Giamarchi and H. J. Schulz, Phys. Rev. B37, 325 (1988).

${ }^{28}$ L. P. Kadanoff, Ann. Phys. 120, 39 (1979); L. P. Kadanoff and A. C. Brown, ibid. 121, 318 (1979.)
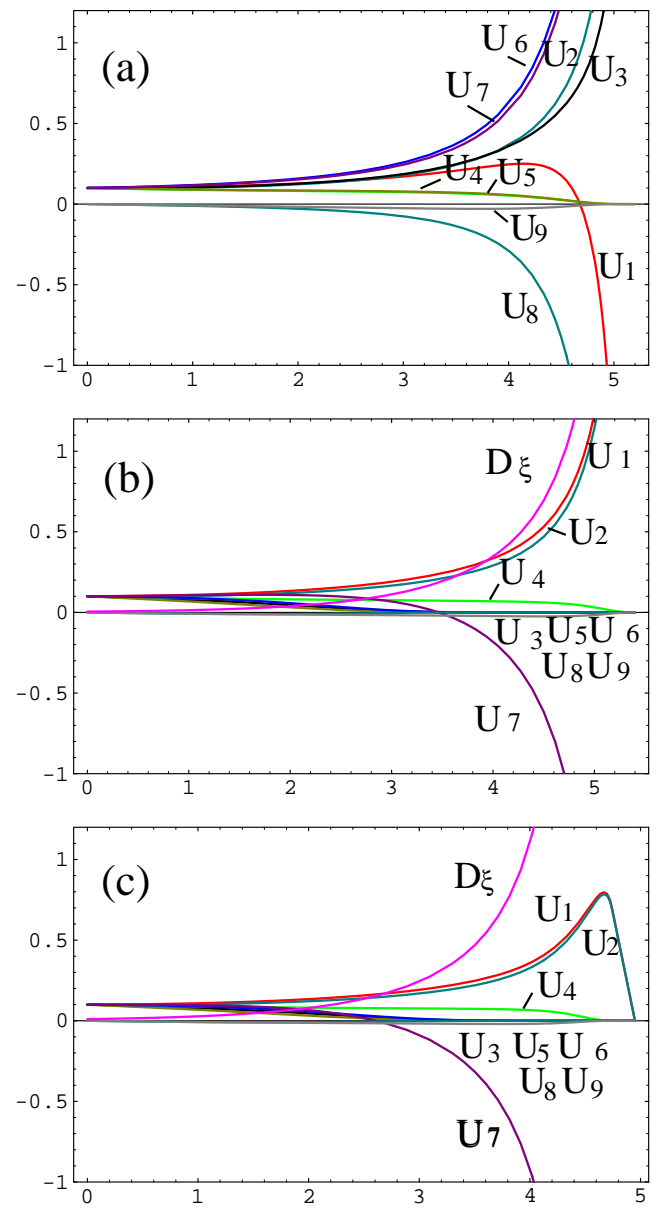

FIG 1. Plots of $\tilde{U}_{i}$ and $\tilde{D}_{\xi}$ vs the scaling parameter $l$ in the case A for different initial values of $\tilde{D}_{\eta \pm}$ and $\tilde{D}_{\xi i}: \quad$ (a) $\tilde{D}_{\eta \pm}(0)=\tilde{D}_{\xi i}(0)=0 . \quad$ (b) $\tilde{D}_{\eta \pm}(0)=0.9$, $\tilde{D}_{\xi i}(0)=0.005$. (c) $\tilde{D}_{\eta \pm}(0)=0.9, \tilde{D}_{\xi i}(0)=0.01$. The initial values of the TL liquid parameters and $\tilde{U}_{i}$ are set as $K_{ \pm \rho}(0)=0.98, K_{+\sigma}(0)=1.0, K_{-\sigma}(0)=0.86$, and $\tilde{U}_{i}(0)=0.1$ for $i=1 \sim 7, \tilde{U}_{8}(0)=\tilde{U}_{9}(0)=0$. As the initial values of $\tilde{D}_{\eta}$ and $\tilde{D}_{\xi i}$ increases, the mass generating terms scale to zero, and the Anderson localized state realizes.

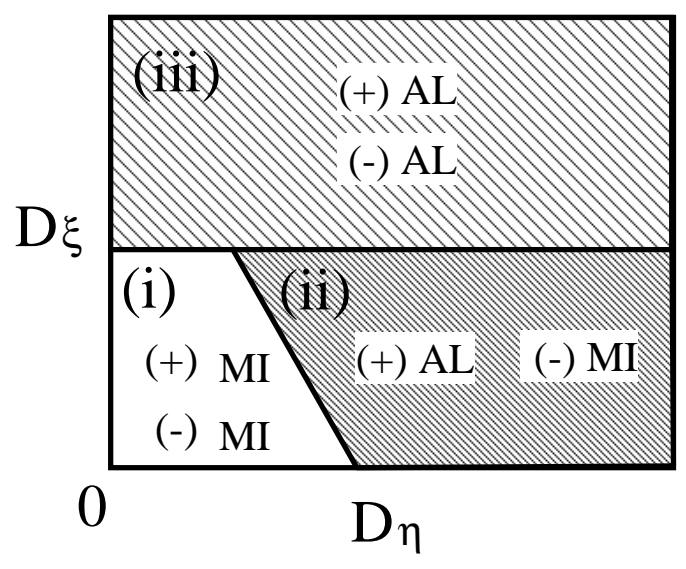

FIG 2. Phase diagram in the $D_{\eta \pm}-D_{\xi i}$ plane. (i) Mott insulator $(\mathrm{MI})$ in both the $(+)$ and $(-)$-charge sectors. (ii) Anderson localization (AL) in the $(+)$-charge sector, and MI in the $(-)$-charge sector. (iii) AL in both the $(+)$ and $(-)$-charge sectors. 

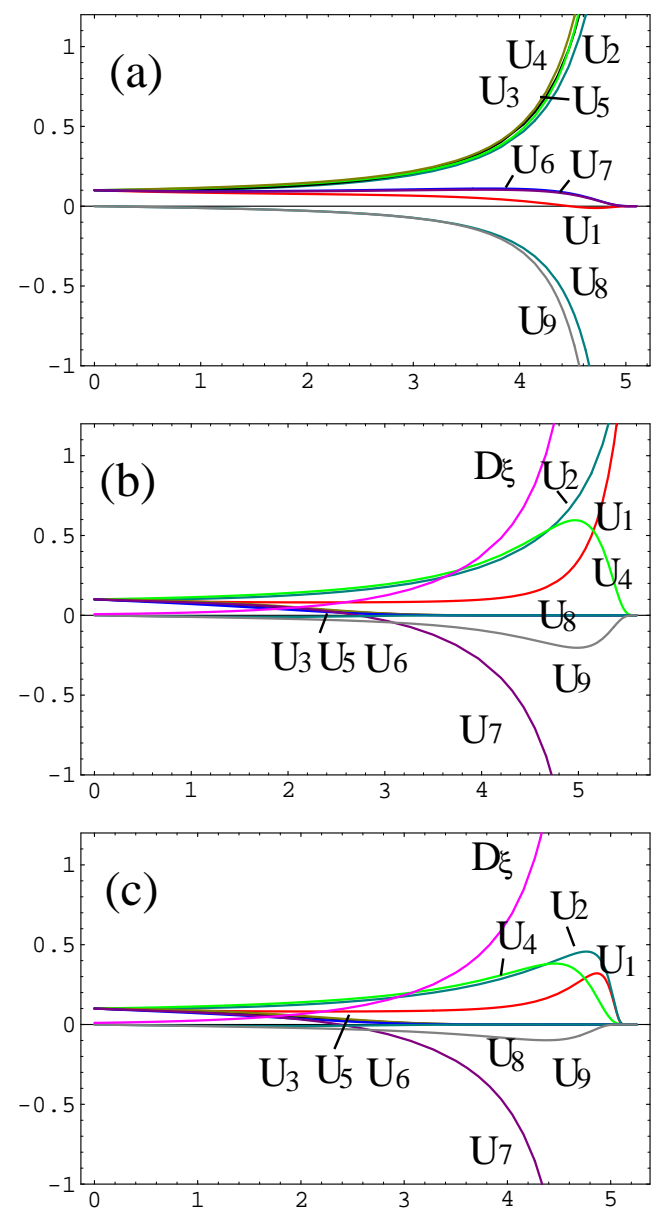

FIG 3. Plots of $\tilde{U}_{i}$ and $\tilde{D}_{\xi}$ vs $l$ in the case B. for the different initial values of $\tilde{D}_{\eta \pm}$ and $\tilde{D}_{\xi i}$ : (a) $\tilde{D}_{\eta \pm}(0)=$ $\tilde{D}_{\xi i}(0)=0 . \quad$ (b) $\tilde{D}_{\eta \pm}(0)=0.9, \tilde{D}_{\xi i}(0)=0.007$. (c) $\tilde{D}_{\eta \pm}(0)=0.9, \tilde{D}_{\xi i}(0)=0.01$. The initial values of the TL parameters and $\tilde{U}_{i}$ are set as $K_{ \pm \rho}(0)=0.98$, $K_{+\sigma}(0)=1.0, K_{-\sigma}(0)=1.1, \tilde{U}_{i}(0)=0.1$ for $i=1 \sim 7$, and $\tilde{U}_{8}(0)=\tilde{U}_{9}(0)=0$. 